\title{
Observation of nano-indent induced strain fields and dislocation generation in Silicon wafers using Micro-Raman Spectroscopy and White Beam X-Ray Topography.
}

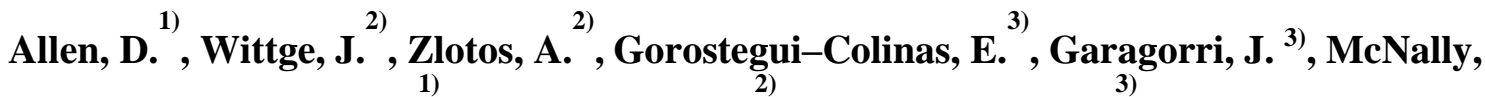 \\ P.J. ${ }^{1)}$, Danilewsky, A.N. ${ }^{2)}$ and Elizalde, M. R.
}

(1) Dublin City University, Research Institute for Networks and Communications Engineering, Dublin 9, Ireland.

(2) University Freiburg, Kristallographie, Gewowiss. Institut, Freiburg, Germany.

(3) Centro de Estudios e Investigaciones Tecnicas de Gipuzkoa, CEIT, San Sebastian, Spain.

\begin{abstract}
:
In the semiconductor manufacturing industry, wafer handling introduces micro-cracks at the wafer edge. During heat treatment these can produce larger, long-range cracks in the wafer which can cause wafer breakage during manufacture. Two complimentary techniques, microRaman spectroscopy ( $\mu \mathrm{RS}$ ) and White Beam Synchrotron X-ray Topography (WBSXRT) were employed to study both the micro-cracks and the associated strain fields produced by nano-indentations in Si wafers, which were used as a means of introducing controlled strain in the wafers. It is shown that both the spatial lateral and depth distribution of these long range strain fields are relatively isotropic in nature. The Raman spectra suggest the presence of a region under tensile strain beneath the indents, which can indicate a crack beneath the indent and the data strongly suggests that there exists a minimum critical applied load below which cracking will not initiate.
\end{abstract}

\section{Introduction:}

The process of utilising nano-indentation in Si to simulate wafer damage has been widely discussed by Cheong, Lawn and others [1-3]. It is known that the indentation of Si can create areas of high stress that results in phase changes to the crystal structure [4] and that in the core region of the indent, where the stresses are highest, the crystalline $\mathrm{Si}$ (c-Si) structure may become unstable and transform into a metallic phase (Si-II) under indentation load, and then 
undergo phase transformation to a metastable state under indentation unload. The final state can be an amorphous phase, a-Si, or a high pressure polymorph (e.g. Si-III/Si-XII) [5, 6].

Micro Raman spectroscopy ( $\mu \mathrm{RS}$ ) is a highly selective and advantageous tool for the investigation of nano-indents in semiconductor wafers [7]. Lowney et al., among others, demonstrated that Raman scattering is a very sensitive technique for the probing of local atomic environments of c-Si [8].

White Beam Synchrotron X-ray topography (WBSXRT) is a non-destructive imaging technique that is widely used for the characterisation of both long range and short range strain in single crystals $[9,10]$. In this technique every diffraction vector $\vec{g}$, which fulfils Bragg's law, results in one topograph. From this both the Burgers vector $\vec{b}$ and magnitude of the vector $|b|$ can be calculated from the expressions:

$\vec{b} \cdot \vec{g}=0 \quad(1)$

$|b|=\frac{a}{2} \sqrt{h^{2}+k^{2}+l^{2}}$

where $a$ is the unit cell length and $\mathrm{h}, \mathrm{k}$ and $\mathrm{l}$ are the Bravais-Miller indices. During one exposure a Laue pattern of topographs is captured on photographic film [10]. These can be used to study the both the short and long range strain fields within the silicon sample.

Defined defects, i.e. nano-indents, were introduced into silicon (100) samples using a Nanoindenter II from Agilent equipped with a sharp Berkovich tip. Numerous indented samples were produced, including samples with a $5 \times 5$ array. By way of example, figure 1 shows a $02 \overline{2}$ large area transmission (LAT) topograph, and figure 2 shows a $6 \overline{2} 0$ large area back reflection topgraph (LABRT) of the same indents, showing the strain field induced contrast, associated with the indents. The array was produced using vertical applied forces of $600 \mathrm{mN}$, $500 \mathrm{mN}, 400 \mathrm{mN}, 300 \mathrm{mN}, 200 \mathrm{mN}$ and $100 \mathrm{mN}$, respectively, at a load/unload rate of $10 \mathrm{mN} / \mathrm{s}$, 
the indents being $100 \mu \mathrm{m}$ apart. Other samples were produced with a single line of 15 indents each $1 \mathrm{~mm}$ apart.

The indents were also studied using micro-Raman Spectroscopy. This technique confirmed the presence of numerous phases of silicon; both $\mathrm{Si}-\mathrm{XII}$ and $\mathrm{Si}$-III are produced by the indentation process and both compressive and tensile strain exists in the indented Si.

The indents were typically observed using both large area transmission and back reflection xray topography. LABRT, with X-ray penetration depths $\sim 10-50 \mu \mathrm{m}$, was used to characterise the strain fields surrounding the indents near the surface of the Si wafers.

\section{Experimental Setup:}

In this study c-Si wafers with (100) surface crystallographic orientation were used. A series of nano-indents were produced as previously described. The indents were then studied and the Raman spectra were recorded with a Jobin-Yvon LabRam HR800 Raman microscope.

Each Raman spectrum was acquired as three accumulations of 10s each. A grating of 2,400 grooves/mm was used with the exciting laser source being focused through an Olympus ${ }^{\circledR}$ 100x microscope objective for the $488 \mathrm{~nm} \mathrm{Ar}^{+}$laser and an $\mathrm{OFR}^{\circledR}$ Near Ultraviolet (NUV) 40x lens for the $325 \mathrm{~nm}$ He-Cd laser. Holographic edge and notch filters reject the Rayleigh light and an interference filter rejects the plasma lines of the laser. The laser spot size was focused to $\sim 1 \mu \mathrm{m}$ in diameter for the $\mathrm{Ar}^{+}$laser and $\sim 0.8 \mu \mathrm{m}$ in diameter for the He-Cd laser. The probe depth $\left(d_{p}\right)$ of the lasers is calculated from [11]:

$d_{p}=2.3 / 2 \alpha$

where $\alpha$ is the photo absorption coefficient of silicon [12]. The $d_{p}$ for the $\operatorname{Ar}^{+}$laser is $\sim 570$ $\mathrm{nm}$ while that of the He-Cd laser is $\sim 9 \mathrm{~nm}$. Probing with the He-Cd laser can, therefore, reveal the stress regime and material structure near the silicon surface [13]. 
The indents were then studied using WBSXRT, at the ANKA Synchrotron, Institute for Synchrotron Studies, Forschungszentrum Karlsruhe, Germany, and at the HASYLAB Synchrotron, Deutsches Elektronen-Synchrotron DESY, Hamburg, Germany, in both transmission and back reflection modes. The X-ray film upon which a full Laue pattern of the crystal is collected is Slavich VRP-M film with a grain size of $\sim 0.04 \mu \mathrm{m}$ and the beam can be collimated both vertically and laterally from $2 \mu \mathrm{m}$ to $10 \mathrm{~mm}$ in $2.5 \mu \mathrm{m}$ steps with sub- $\mu \mathrm{m}$ reproducibility [14]. The ANKA beam line has a white beam energy range of $\sim 1.5 \mathrm{keV}$ to 33 $\mathrm{keV}$ that is sourced from a $1.5 \mathrm{~T}$ bending magnet. The beam line is fed from a $2.5 \mathrm{GeV}$ storage ring [15], while the HASYLAB beam line has a white beam energy range of $\sim 5 \mathrm{keV}$ to $41 \mathrm{keV}$ sourced from a $1.218 \mathrm{~T}$ bending magnet. The beam line is fed from a $4.45 \mathrm{GeV}$ storage ring [23].

\section{Results and Discussion:}

Any applied mechanical stress or strain in Silicon affects the frequencies of the Raman bands and lifts their degeneracy $[16,17]$. In the absence of strain, the first order Stokes Raman spectrum of silicon shows a peak which corresponds to the triply degenerate Transverse Optical (TO) phonon and has a wave number shift, with respect to the excitation light source, of $\sim 520 \mathrm{~cm}^{-1}[17]$. Assuming the strain in the indented silicon to be biaxial, the stress components $\sigma_{x x}$ and $\sigma_{y y}$ are equal and can be estimated from [17]:

$\sigma_{x x}=\sigma_{y y}=\frac{\Delta \omega}{-4} G P a$

where $\Delta \omega\left(\mathrm{cm}^{-1}\right)=\omega_{1}-\omega_{0}, \omega_{0}$ is the Si-Si TO unstrained Raman spectral line at $520 \mathrm{~cm}^{-1}$ and $\omega_{1}$ is the measured Raman spectral line of the sample. Figures $3 \mathrm{a}$ and $3 \mathrm{~b}$ show the strain data for the indents studied. Strain is displayed on the left hand side of the figure in $G P a$, relative wavenumbers are on the right hand side in $\mathrm{cm}^{-1}$ and the bottom of the graph shows distance along the Si surface with the edges of the indents marked by a two vertical lines. Compressive 
strain (C), i.e. blueshift of the TO mode, is marked with a blue (up) arrow while tensile strain (T), Raman mode redshifted, is marked with a red (down) arrow. Figure 3a shows the data from the $600 \mathrm{mN}, 500 \mathrm{mN}$, and $400 \mathrm{mN}$ indents while figure $3 \mathrm{~b}$ shows data from the $300 \mathrm{mN}$, $200 \mathrm{mN}$ and $100 \mathrm{mN}$ indents, the data having been taken with the $498 \mathrm{~nm} \mathrm{Ar}^{+}$laser. The data shows that all the indents, bar the $100 \mathrm{mN}$ indent, progress from compressive strain, at the edge of the indent, to tensile strain in the centre, and back to compressive strain at the other edge. Lawn et al. [18] postulated that a sharp indenter, such as a Berkovich tip, will cause the formation of an elastic/plastic field beneath the area of indentation, figure 4 . If the base of the plastic zone, the area of maximum tension, intercepts a nucleation centre then a crack can be initiated. This type of crack was observed by both Lawn et al. [18] and Lloyd et al. [19]. A characteristic of sharp indenters is that they can generate their own flaws within initially defect free material [19] and there exists a critical loading below which a crack will not initiate. No tensile shift was observed in the $100 \mathrm{mN}$ indent, which indicates the likelihood of a minimum threshold pressure required to initiate a crack. Further experimentation is ongoing to prove this hypothesis. A typical example of the crack development is shown in Transmission Electron Microscopy (TEM) micrographs performed on a $200 \mathrm{mN}$ indent (see figure 5), wherein the cracks are $\sim 160 \mathrm{~nm}$ below the indent. At this depth the resulting tensile strain field does not impact upon the upper surface regions, where only compressive strain should be observed. This agrees with the data obtained from the $325 \mathrm{~nm} \mathrm{He}-\mathrm{Cd}$ laser. The probe photons only penetrate to $\sim 9 \mathrm{~nm}$ and the resultant data not shown here, show no evidence of tensile strain.

High pressure polymorphs of silicon have also been observed within the indented Si. The phases are primarily Si-III and Si-XII [2, 3] (see figures 6a and 6b). These phases were observed in all the indents except for the $100 \mathrm{mN}$ indents (figure 6c). Figure $6 \mathrm{c}$ also shows the calibration spectrum obtained from unstrained silicon showing a sharp peak at $520 \mathrm{~cm}^{-1}$, all measurements having been taken using the $\operatorname{Ar}^{+}$laser, $d_{p} \sim 570 \mathrm{~nm}$. 
It is known that the polymorph states can only form from the $\beta$-Sn phase ( $\mathrm{Si}-\mathrm{II})$ [20] and the lack of these polymorphs indicates that the applied pressure did not reach the $8.8 \mathrm{GPa}$ required to initiate the $\mathrm{Si}-\mathrm{I}$ to $\mathrm{Si}-\mathrm{II}$ transformation [21].

The LABRT images, figure 2, of the indents show the spatial distribution of the long range lateral strain fields to be relatively isotropic in nature, and the isotropy appears to be independent of the applied pressure.

Figure 7 shows an example of the finite element strain simulation of a $250 \mathrm{mN}$ indent showing von Mises strain in $\mathrm{mPa}$. The simulation software is limited in resolution and, at present, cannot match that of the topography results which have a spatial resolution of $\sim 4 \mu \mathrm{m}$ [24]. One consequence of this is that the strain fields in the simulation appear more compressed than the corresponding field shown in fig. 2 , however the structure of the lateral strain field appears to be consistent with that seen from LAT. The structure of the strain fields, both lateral and depth is also consistent with the section transmission image shown in fig. 9. This figure is taken from a $3 \mathrm{D}$ rendering of the section images.

These simulations were produced using Abaqus/C.A.E. v.6.7-1 simulation software, run on an Intel ${ }^{\circledR}$ Core $^{\mathrm{TM}} 2$ Quad processor. The simulation used a mesh size of $48 \times 96 \times 48 \mu \mathrm{m}$ with a uniform element size of $0.25 \mu \mathrm{m}$ in the indent area $(8 \times 16 \times 3 \mu \mathrm{m})$, the element size getting progressively larger towards the wafer edges, giving a total of 60,416 elements.

From the scanning electron microscope (SEM) image, figure 8, slip bands can be observed emanating from the indents prior to any thermal treatment. These slip directions are [110] which indicates that they are shear type as their source lies at the tips of the indent. This also suggests a plane strain condition surrounding the indents and strongly suggests the formation of a plastic zone within the silicon due to the indent process [24]. This type of dislocation structure is also thought to increase the fracture toughness of the crystal [25] though this needs to be verified. 


\section{Conclusion:}

In order to replicate wafer handling damage silicon wafers were nano-indented with a Berkovich tip and studied using $\mu \mathrm{RS}$ and WBSXRT. Both techniques show the relatively isotropic spatial lateral strain fields surrounding the indents which correlate well with finite element strain simulations. TEM images confirm the presence of both lateral and median cracks in the bulk silicon below the indents, which also correlates with theory and strain data obtained from $\mu \mathrm{RS}$. Theory and data also suggest that there is a minimum critical loading below which cracking will not initiate.

\section{References:}

[1] W. C. D. Cheong and L. C. Zhang, J. Mater. Sci. Lett. 19, 439 (2000).

[2] B. R. Lawn, J. Mater. Res. 19, 22 (2004).

[3] B. R. Lawn, D. B. Marshall, and P. Chantikul, J. Mater. Sci. 16, 1769 (1981).

[4]Y. Gogotsi, A. Kailer, and K. G. Nickel, Mat. Res. Innovat. 1, (1997) 3.

[5] R. Rao, J. E. Bradby, S. Ruffell, and J. S. Williams, J. Microelec. 38, (2007) 722.

[6] A. Kailer, K. G. Nickel, and Y. Gogotsi, J. Raman Spec. 30, (1999) 939.

[7] D. C. Mahon, P. J. Mahon, and D. C. Creagh, Nuc. Instrum. Meth. Phys. Res. 580, (2007) 430.

[8] D. Lowney, T. S. Perova, M. Nolan, P. J. McNally, R. A. Moore, H. S. Gamble, T. Tuomi, R. Rantamaki, and A. N. Danilewsky, Semicond. Sci. Tech. 17, (2002) 1081.

[9] A. N. Danilewsky, A. Rack, J. Wittge, T. Weitkamp, R. Simon, H. Riesemeier, and T. Baumbach, Nuc. Instrum. Meth. Phys. Res. 266, (2008) 2035.

[10] A. N. Danilewsky, R. Simon, A. Fauler, M. Fiederle, and K. W. Benz, Nuc. Instrum. Meth. Phys. Res. 199 , (2003) 71.

[11] J. Takahashi and T. Makino, J. Appl. Phys. 63, (1988) 87.

[12] D. E. Aspnes and A. A. Studna, Phys. Rev. B 27, (1983) 985.

[13] K. F. Dombrowski, B. Dietrich, I. De Wolf, R. Rooyackers, and G. Badenes, Micro. Rel. 41, (2001) 511.

[14] R. Simon and A. N. Danilewsky, Nuc. Instrum. Meth. Phys. Res. 199, (2003) 550. 
[15] M. Cholewa and A. Rack, ANKA Instrumentation Book (ANKA Angstroemquelle Karlsruhe, Germany, 2007), p. 84.

[16] Y. Kang, Y. Qiu, Z. Lei, and M. Hu, Opt. Las. in Eng. 43, (2005) 847.

[17] I. De Wolf, Semicond. Sci. Tech. 11, (1996) 139.

[18] B. R. Lawn and A. G. Evans, J. Mater. Sci. 12, (1977) 2195.

[19] S. J. Lloyd, J. M. Molina-Aldareguia, and W. J. Clegg, J. Mater. Res. 16, (2001) 3347.

[20] R. J. Needs and R. Martin, Phys. Rev. B 30, (1984) 5390.

[21] J. Z. Hu and I. L. Spain, Solid State Commun. 51, (1984) 263.

[22] B. R. Lawn, J. Mater. Res. 19, 22 (2004).

[23] W. Drube and W. Laasch, Photon Science 2008. Highlights and HASYLAB Annual Report (Heigener Europrint, Germany, 2008).

[24] M. Tanaka, K. Higashida, and T. Haraguchi, Mat. Sci. Eng. A 387-389, 433 (2004).

[25] M. Tanaka and K. Higashida, J Electron Microsc (Tokyo) 53, 353 (2004).

Acknowledgment: Financial support by EU-FP7 project no. 216382 SIDAM is gratefully acknowledged.

This work was part-funded by the Irish Higher Education Authority PRTLI "INSPIRE" project

This work was supported by the European Community—Research Infrastructure Action under the FP7 "Structuring the European Research Area" program. 


\section{Figure Captions:}

Figure 1: $02 \overline{2}$ LAT topograph of a typical $5 \times 5$ array of nano-indents.

Figure 2: Example of $6 \overline{2} 0$ LABRT of a typical $5 \times 5$ array of nano-indents

Figure 3a: $\mu \mathrm{RS}$ strain data for $600 \mathrm{mN}, 500 \mathrm{mN}$ and $400 \mathrm{mN}$ indents. Probed with an Ar+ laser, $d_{p} \sim 570 \mathrm{~nm}$.

Figure 3b: $\mu \mathrm{RS}$ strain data for $300 \mathrm{mN}, 200 \mathrm{mN}$ and $100 \mathrm{mN}$ indents. Probed with an $\mathrm{Ar}^{+}$ laser, $\mathrm{d}_{\mathrm{p}} \sim 570 \mathrm{~nm}$.

Figure 4: Model for median crack initiation in an elastic/plastic indent. (a shows the indenter, $\mathrm{b}$ the plastic field and $\mathrm{c}$ the nucleation centre.)[15]

Figure 5: TEM image of a $200 \mathrm{mN}$ indent showing cracks.

Figure 6a: Raman spectra from $600 \mathrm{mN}, 500 \mathrm{mN}$ and $400 \mathrm{mN}$ indents. Data taken from the centre of the indent and probed with an $\mathrm{Ar}^{+}$laser, $\mathrm{d}_{\mathrm{p}} \sim 570 \mathrm{~nm}$.

Figure 6b: Raman spectra from 300mN and $200 \mathrm{mN}$ indents. Data taken from the centre of the indent and probed with an $\mathrm{Ar}^{+}$laser, $\mathrm{d}_{\mathrm{p}} \sim 570 \mathrm{~nm}$.

Figure 6c: Raman spectra from 100mN indents and calibration spectrum from unstrained Si. Data taken from the centre of the indent and probed with an $\mathrm{Ar}^{+}$laser, $\mathrm{d}_{\mathrm{p}} \sim 570 \mathrm{~nm}$.

Figure 7: Finite element simulation of a $250 \mathrm{mN}$ indent.

Figure 8: SEM image of an indent showing slip bands emanating from the indent tips 
Figure 9: 3D model of a section topograph of a $250 \mathrm{mN}$ indent 